\title{
The plasma membrane channel ORAl1 mediates detrimental calcium influx caused by endogenous oxidative stress
}

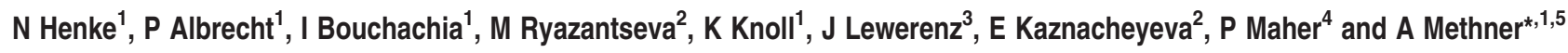

The mouse hippocampal cell line HT22 is an excellent model for studying the consequences of endogenous oxidative stress. Addition of extracellular glutamate depletes the cells of glutathione (GSH) by blocking the glutamate - cystine antiporter system $x_{c}^{-}$. GSH is the main antioxidant in neurons and its depletion induces a well-defined program of cell death called oxytosis, which is probably synonymous with the iron-dependent form of non-apoptotic cell death termed ferroptosis. Oxytosis is characterized by an increase of reactive oxygen species and a strong calcium influx preceding cell death. We found a significant reduction in store-operated calcium entry (SOCE) in glutamate-resistant $\mathrm{HT} 22$ cells caused by downregulation of the $\mathrm{Ca}^{2+}$ channel ORAl1, but not the $\mathrm{Ca}^{2+}$ sensors STIM1 or STIM2. Pharmacological inhibition of SOCE mimicked this protection similarly to knockdown of ORAl1 by small interfering RNAs. Long-term calcium live-cell imaging after induction of the cell death program showed a specific reduction in $\mathrm{Ca}^{2+}$-positive cells by ORAl1 knockdown. These results suggest that dysregulated $\mathrm{Ca}^{2+}$ entry through ORAl1 mediates the detrimental $\mathrm{Ca}^{2+}$ entry in programmed cell death induced by GSH depletion. As this detrimental $\mathrm{Ca}^{2+}$ influx occurs late in the course of the cell death program, it might be amenable to therapeutic intervention in diseases caused by oxidative stress.

Cell Death and Disease (2013) 4, e470; doi:10.1038/cddis.2012.216; published online 24 January 2013

Subject Category: Neuroscience

Oxidative stress is a phenomenon involved in a broad range of pathological conditions of the nervous system such as Alzheimer's and Parkinson's disease, stroke and trauma. It is provoked by an imbalance between superoxide production in the mitochondria and insufficient clearance of reactive oxygen species (ROS) through antioxidative defense mechanisms of the cell. One major defense pathway of nerve cells against ROS depends on the production of glutathione $(\mathrm{GSH})$, a tripeptide composed of glutamate, cysteine and glycine, via the enzymes glutamate-cysteine ligase and GSH synthetase. In its reduced state, GSH serves as electron donor to detoxify ROS and is thereby oxidized to form GSH disulfide. The recovery of $\mathrm{GSH}$ from $\mathrm{GSH}$ disulfide is mediated by GSH reductase under NADPH consumption. ${ }^{1}$

GSH depletion serves to study the effects of endogenous oxidative stress and can be excellently studied in vitro by treating cells with glutamate, which inhibits cystine uptake through the glutamate/cystine antiporter system $x_{c}^{-}$. Within the cell, cystine is rapidly converted to cysteine, the ratelimiting amino acid for GSH synthesis. Cystine deprivation then causes secondary GSH depletion and a programmed cell death by oxytosis or oxidative glutamate toxicity, which is clearly distinct from apoptosis, necrosis, and cell death associated with autophagy, but probably synonymous with the recently described iron-dependent form of non-apoptotic cell death termed ferroptosis, which seems to be involved in the selective elimination of some tumor cells and protection from neurodegeneration. ${ }^{2}$

A well-established model system for oxytosis/ferroptosis is glutamate-induced cell death in the hippocampal cell line HT22, which has been used extensively to clarify the cascade leading to cell death and to identify antioxidant pathways and

\footnotetext{
${ }^{1}$ Department of Neurology, Heinrich-Heine Universität Düsseldorf, Düsseldorf, Germany; ${ }^{2}$ Institute of Cytology St. Petersburg, St. Petersburg, Russia; ${ }^{3}$ University of Ulm, Department of Neurology, Ulm, Germany; ${ }^{4}$ Cellular Neurobiology Laboratory, Salk Institute for Biological Studies, La Jolla, CA, USA and ${ }^{5}$ Focus Program Translational Neuroscience (FTN), Rhine Main Neuroscience Network $\left(\mathrm{rmn}^{2}\right)$, Johannes Gutenberg University Medical Center Mainz, Department of Neurology, Mainz, Germany

${ }^{*}$ Corresponding author: Professor A Methner, Focus Program Translational Neuroscience (FTN), Rhine Main Neuroscience Network (rmn²), Johannes Gutenberg University Medical Center Mainz, Department of Neurology, Langenbeckstr. 1, D-55131 Mainz, Germany, Tel: + 496131172695 , Fax: + 49 6131 17 5967; Email: axel.methner@gmail.com

Keywords: STIM1; SOCE; oxidative stress

Abbreviations: 2-APB, 2-aminoethoxydiphenyl borate; ANOVA, Analysis of variance; BSO, L-buthionine-(S,R) sulfoximine; cGMP, Cyclic guanosine monophosphate; CTB, Cell titer blue; EGTA, Ethylene glycol tetraacetic acid; ER, Endoplasmic reticulum; FCS, Fetal calf serum; GAPDH, Glyceraldehyde 3-phosphate dehydrogenase; GSH, Glutathione; HBSS, Hank's balanced salt solution; HPRT, Hypoxanthine-phosphoribosyltransferase; IP3, Inositol trisphosphate; IP3R, Inositol trisphosphate receptor; iPLA2 $\beta, \mathrm{Ca}^{2+}$-independent phospholipase A2; LOX, Lipoxygenase; MTT, 3-(4,5-Dimethylthiazol-2-yl)-2,5-diphenyltetrazolium bromide; NAC, N-acetyl-Lcysteine; NADPH, Nicotinamide adenine dinucleotide phosphate; PBS, Phosphate-buffered saline; pCPT-cGMP, 8-(4-chlorophenylthio)-guanosine 3',5'-cyclic monophosphate; RFP, Red fluorescent protein; ROS, Reactive oxygen species; SEM, Standard error of the mean; SERCA, Sarcoplasmic/endoplasmic reticulum Ca2 + -ATPase; SOCE, Store-operated calcium entry; STIM1, Stromal interaction molecule 1; STIM2, Stromal interaction molecule 2; TRPM7, Transient receptor potential melastatin 7

Received 4.10.12; revised 29.11.12; accepted 6.12.12; Edited by A Finazzi-Agro'
} 
proteins (reviewed $\mathrm{in}^{3}$ ). In this system, GSH depletion leads to an exponential increase in ROS that mostly originates from mitochondrial complex I activity. ${ }^{4}$ After $\sim 6 \mathrm{~h}$ of glutamate exposure, the lipid-oxidizing enzyme 12/15-lipoxygenase (12/15- LOX; EC 1.13.11.33) is activated and generates 12- and 15- hydroxyeicosatetraenoic acids ${ }^{5}$ that directly damage mitochondria, and cause mitochondrial depolarization and increased ROS production. ${ }^{6}$ The eicosanoids produced by 12-LOX are, however, also activators of soluble guanylate cyclases and thereby increase the concentration of intracellular cyclic guanosine monophosphate (cGMP), resulting in a detrimental influx of calcium at the end of the cell death cascade through a yet uncharacterized cGMP-dependent calcium channel. ${ }^{7}$ This $\mathrm{Ca}^{2+}$ influx is essential for the completion of the cell death program, as proven by the fact that glutamate-treated HT22 cells do not die when $\mathrm{Ca}^{2+}$ influx is blocked by $\mathrm{CoCl}_{2}$ or in $\mathrm{Ca}^{2+}$-free medium, ${ }^{7-9}$ but the molecular identity of the contributing $\mathrm{Ca}^{2+}$ channels is still unknown.

To identify the mechanism of $\mathrm{Ca}^{2+}$ entry in the final phase of oxidative glutamate toxicity, we compared the cellular calcium state of glutamate-sensitive and resistant HT22 cells, which are resistant due to the increased expression of various proteins with antioxidant properties, ${ }^{10-13}$ and found an isolated attenuation of store-operated calcium entry (SOCE) in the resistant cells. SOCE is activated when the endoplasmic reticulum (ER), the main cellular calcium store, is depleted, for example,. during inositol trisphosphate (IP3)mediated signaling events. When a membrane receptor is activated by its ligand, IP3 is generated near the plasma membrane and quickly diffuses through the cytoplasm to reach its receptor (Inositol trisphosphate receptor, IP3R) at the ER membrane. Binding of IP3 to IP3R releases $\mathrm{Ca}^{2+}$ stored in the ER lumen and generates a cytosolic $\mathrm{Ca}^{2+}$ signal, resulting in $\mathrm{ER} \mathrm{Ca}^{2+}$-store depletion. To refill the $\mathrm{ER}$, plasma membrane $\mathrm{Ca}^{2+}$ channels need to be activated to permit $\mathrm{Ca}^{2+}$ entry from the extracellular space, so-called SOCE. The molecule that transmits the information of $\left[\mathrm{Ca}^{2+}\right]_{\mathrm{ER}}$ to plasma membrane $\mathrm{Ca}^{2+}$ channels is stromal interaction molecule 1 (STIM1). ${ }^{14,15}$ STIM1 clusters into punctae close to the plasma membrane upon store depletion and binds and activates ORAI1, a plasma membrane calcium channel. ${ }^{16-18}$

In this study, we provide evidence that dysregulated SOCE through ORAl1 is the main calcium entry mechanism during oxidative glutamate toxicity, suggesting that SOCE inhibition might be a valuable tool in the treatment of diseases associated with increased oxidative stress.

\section{Results}

\section{Reduced store-operated $\mathrm{Ca}^{2+}$ entry in hippocampal} cells resistant to oxidative stress. Glutamate-resistant HT22 cells are protected against oxidative glutamate toxicity (Figure 1a) mainly via an elevated GSH content due to an increased expression of the glutamate/cystine antiporter subunit $\mathrm{xCT}{ }^{19}$ They also show an increased expression of other proteins with antioxidant properties. ${ }^{10,11,13} \mathrm{We}$ therefore reasoned that the function and expression of the sought-after detrimental $\mathrm{Ca}^{2+}$ channel might be altered in these cells and studied the content of the main cellular
$\mathrm{Ca}^{2+}$ store, the $\mathrm{ER}$, by staining the cells with the ratiometric $\mathrm{Ca}^{2+}$-sensitive dye Fura2 followed by treatment with the irreversible sarcoplasmic/ER $\mathrm{Ca}^{2+}$-ATPase (SERCA) inhibitor thapsigargin, which resulted in the expected biphasic cytosolic $\mathrm{Ca}^{2+}$ rise. Although HT22S and R cells behaved similarly during the first phase of $\mathrm{Ca}^{2+}$ rise, which corresponds to $\mathrm{Ca}^{2+}$ passively leaking out of the ER, the second phase of cytosolic $\mathrm{Ca}^{2+}$ rise was remarkably reduced in HT22R compared with HT22S cells (Figure 1b).
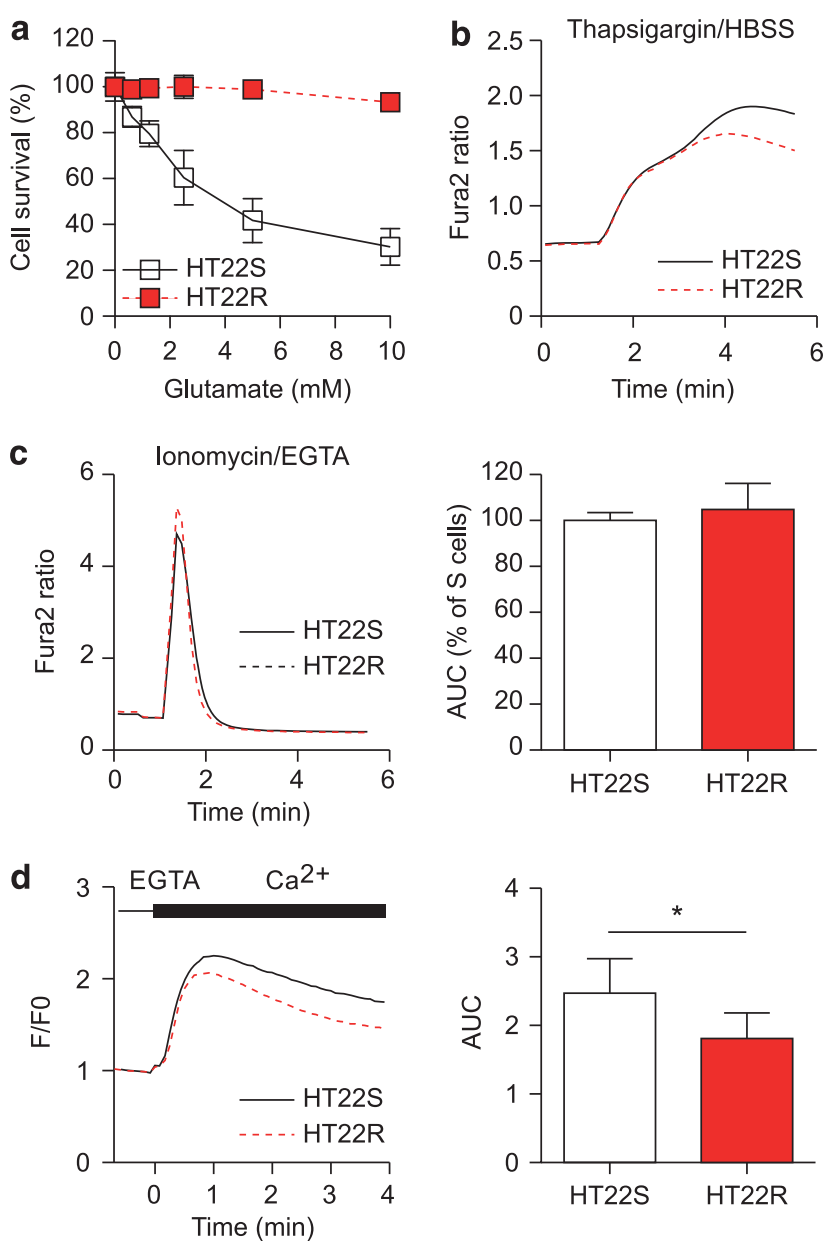

Figure 1 Reduced store-operated $\mathrm{Ca}^{2+}$ in hippocampal cells resistant to oxidative stress. (a) 5000 glutamate-sensitive and -resistant HT22S and R cells were seeded into 96-well plates and subjected to the indicated concentrations of glutamate $24 \mathrm{~h}$ later; viability was quantitated again $16 \mathrm{~h}$ later with the cell-titer blue reagent. Mean \pm S.E.M. fluorescence values of three replicates obtained in three independent experiments were plotted against the glutamate concentration. $(\mathbf{b}-\mathbf{d})$ Fura2-live-cell imaging of glutamate-sensitive and -resistant HT22S and R cells. (b) After $1 \mathrm{~min}$ of baseline recording in HBSS, $2 \mu \mathrm{m}$ of thapsigargin was added and the fura2 signal recorded for an additional $4 \mathrm{~min}$. (c) After $30 \mathrm{~s}$ of baseline recording in HBSS with $\mathrm{Ca}^{2+}$, EGTA was added. After a further $30 \mathrm{~s}, 5 \mu \mathrm{m}$ of ionomycin was applied and the fura2 signal recorded for the following $4 \mathrm{~min}$. (d) For measurement of SOCE, ER $\mathrm{Ca}^{2+}$ stores of HT22S and R cells were depleted by incubation in EGTA buffer supplemented with $2 \mu \mathrm{m}$ thapsigargin. After $6 \mathrm{~min}$ of store depletion, the buffer was exchanged with HBSS supplemented with $1.26 \mathrm{~mm} \mathrm{Ca}^{2+}$ and SOCE was recorded. Graph shows only $\mathrm{Ca}^{2+}$ readdition after store depletion. Graphs $1 \mathrm{~b}$ and $c$ show mean fura2 ratio of $20-30$ individually-measured wells for each condition, containing altogether $2000-3000$ cells. Graph $1 \mathrm{~d}$ is pooled from nine wells for each condition containing $>260$ cells. For $C$ and $D$, the area under the curve was calculated and plotted as mean \pm S.E.M. in bar graphs. ${ }^{*} P<0.05$ 

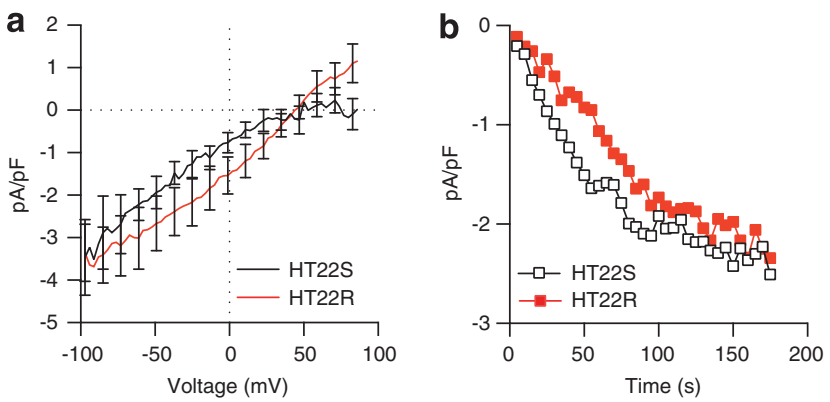

Figure 2 Different SOCE current characteristics in HT22S and glutamateresistant HT22R cells. (a) Average I/V relationships for currents evoked by passive depletion of $\mathrm{Ca}^{2+}$ stores with $1 \mu \mathrm{m}$ thapsigargin in HT22S (black, $n=6$ ) and HT22R (red, $n=11$ ) cells. The I $/ V$ relationships were measured when the currents reached the maximum. The data are shown as mean \pm S.E.M., $P<0.05$. (b) The average amplitude of SOCE currents recorded in whole-cell experiments is shown as a function of time after application of $1 \mu \mathrm{m}$ thapsigargin to HT22S (black, $n=6$ ) and HT22R (red, $n=11$ ) cells. The current amplitudes were measured at each ramp at $-80 \mathrm{mV}$ test potentials. The data are shown as mean

We hypothesized that this second plateau corresponds to reduced SOCE and decided to delve further into this.

To distinguish between $\mathrm{Ca}^{2+}$ leaking from the $\mathrm{ER}$ and $\mathrm{Ca}^{2+}$ entering from outside the cell, we measured the whole-cellular $\mathrm{Ca}^{2+}$ store content and SOCE separately. Fura2-based $\mathrm{Ca}^{2+}$ live-cell imaging yielded no significant difference between HT22S and HT22R in the cytosolic $\mathrm{Ca}^{2+}$ rise induced by treatment with the $\mathrm{Ca}^{2+}$ ionophore ionomycin, which releases the whole-cellular $\mathrm{Ca}^{2+}$ content from the intracellular stores (Figure 1c). For measurement of SOCE activity, we depleted the $\mathrm{ER} \mathrm{Ca}^{2+}$ store by addition of thapsigargin in EGTA buffer. After $6 \mathrm{~min}$ of store depletion, $\mathrm{Ca}^{2+}$ was readded and $\mathrm{Ca}^{2+}$ influx corresponding to SOCE was measured. In line with the data shown in Figure 1b, SOCE activity was significantly reduced in HT22R cells (Figure 1d).

To verify the reduced SOCE capacity of HT22R cells at the single cell level, we assessed SOCE activity by electrophysiological recordings using the whole-cell patch-clamp technique. Currents were evoked by application of $1 \mu \mathrm{m}$ thapsigargin to achieve ER store depletion. Consistent with our results obtained in fura2 life - cell imaging experiments, HT22S and HT22R cells demonstrated different SOCE current - voltage (I/V) relation (Figure 2a). SOCE currents in HT22S cells revealed an inward rectification as expected for I CRAC currents provided by ORAI1 channels, whereas SOCE currents in HT22R cells demonstrated a linear I/V relation with a reversal potential around $+40 \mathrm{mV}$. There was no difference in amplitude or speed of SOCE currents development in time in HT22S and HT22R cells (Figure 2b). These data are in line with an altered composition or abundance of $\mathrm{I}_{\mathrm{CRAC}}$ channels in HT22R cells. We therefore set out to investigate the role of SOCE during oxidative glutamate toxicity.

Prolonged oxidative stress reduces the $\mathrm{ER} \mathrm{Ca}^{2+}$ content and SOCE activity. Glutamate-resistant HT22R cells were continuously cultivated in medium containing $10 \mathrm{~mm}$ glutamate to preserve their resistant phenotype. To test whether glutamate directly or indirectly influences SOCE capacity, we aimed to measure SOCE activity in glutamate-treated HT22S cell. We also hypothesized that cytosolic cGMP might influence the SOCE capacity similarly to glutamate because the $\mathrm{Ca}^{2+}$ influx leading to cell death by oxytosis is preceded by a cytosolic increase of cGMP. ${ }^{7}$ Application of 8-(4chlorophenylthio)-guanosine $3^{\prime}, 5^{\prime}$-cyclic monophosphate (pCPT-cGMP), a cell-permeable cGMP analog, indeed induced death of HT22 cells in a concentration-dependent manner (Figure 3a) and opened plasma membrane $\mathrm{Ca}^{2+}$ channels resulting in a slow but pronounced cytosolic $\mathrm{Ca}^{2+}$ rise, which we measured with the genetically-encoded $\mathrm{Ca}^{2+}$ sensor GCaMP5, expressed from a bicistronic transcript together with the red fluorescent protein (RFP), to normalize the GCaMP5 signal to the level of transfection (Figure 3b).

We elicited SOCE by store depletion through thapsigargininduced SERCA inhibition under $\mathrm{Ca}^{2+}$-free conditions and subsequent restitution of $\mathrm{Ca}^{2+}$. After $8 \mathrm{~h}$ of incubation with either glutamate or PCPT-cGMP, HT22S cells showed a reduced $\left[\mathrm{Ca}^{2+}\right]_{E R}$ and reduced SOCE (Figure 3c). Addition of pCPT-cGMP or glutamate directly at the onset of measurement had no effect (Figure 3d). We therefore hypothesized that this reduction in $\left[\mathrm{Ca}^{2+}\right]_{\mathrm{ER}}$ and SOCE after induction of the cell death program might be a consequence of the endogenous oxidative stress that eventually kills the cells. And indeed, preventing oxidative stress with the antioxidant $\mathrm{N}$-acetyl-L-cysteine (NAC) protected glutamate-treated cells from cell death (Figure $3 e$ ), and partially rescued the $\mathrm{ER} \mathrm{Ca}^{2+}$ content as well as the SOCE capacity after $8 \mathrm{~h}$ of treatment (Figure 3f).

These results were of course not in line with our hypothesis that increased SOCE mediates the cytotoxic $\mathrm{Ca}^{2+}$ influx at the end of the cascade leading to cell death. We reasoned that the cell death program, which is already irreversible after $8 \mathrm{~h}$ of glutamate or 8-pCPT-cGMP treatments, alters many aspects of the intracellular $\mathrm{Ca}^{2+}$ homeostasis, which might obscure the effects on SOCE. We therefore decided to study the effect of SOCE on glutamate- and CGMP-induced cell death pharmacologically.

Pharmacological inhibition of SOCE protects against oxidative stress. We treated HT22 cells with a pharmacological inhibitor of SOCE, 2-aminoethoxydiphenyl borate (2APB), and induced oxidative stress by addition of glutamate. 2-APB concentration-dependently protected against glutamate toxicity (Figure $4 \mathrm{a}$ ) even when added up to $8 \mathrm{~h}$ after glutamate addition (Figure $4 \mathrm{~b}$ ). We also tested the ability of 2-APB to protect from pCPT-cGMP-induced cell death and observed a remarkable protective effect of 2-APB under these conditions, which was even stronger than that of the general $\mathrm{Ca}^{2+}$-channel blocker $\mathrm{CoCl}_{2}$ (Figure 4c). As 2-APB is a well-known inhibitor of SOCE and protects HT22 cells from oxidative glutamate toxicity and pCPT-cGMP even when applied several hours after induction of oxidative stress, these observations support the hypothesis that the detrimental, late-onset $\mathrm{Ca}^{2+}$ influx induced by endogenous oxidative stress is mediated via components of the SOCE machinery.

The SOCE $\mathrm{Ca}^{2+}$ influx channel ORAl1 is downregulated in glutamate-resistant HT22R cells. After clarifying that the observed reduction of SOCE in HT22R cells (Figures 1d 

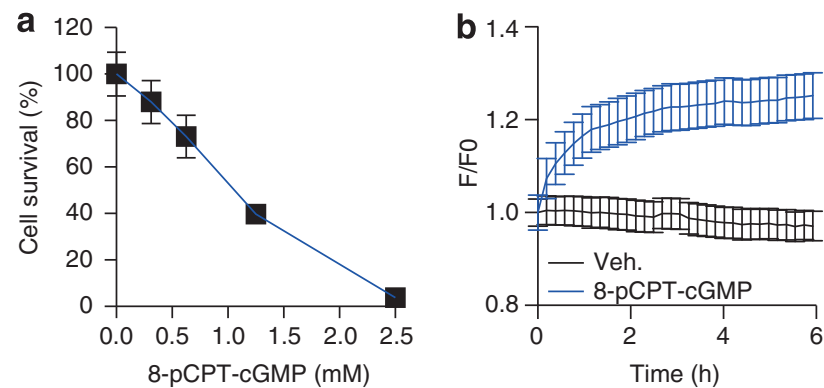

c

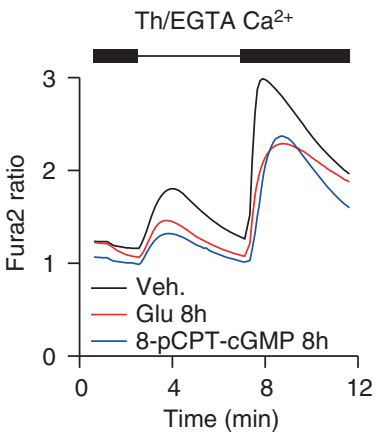

e

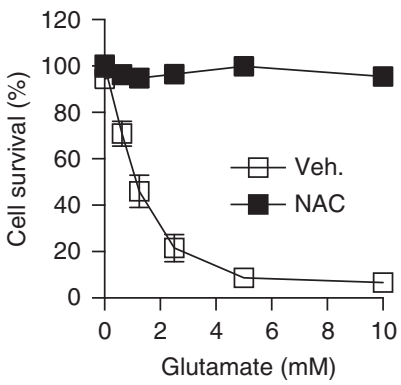

d

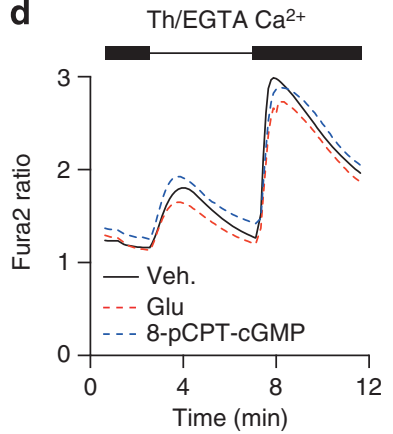

f

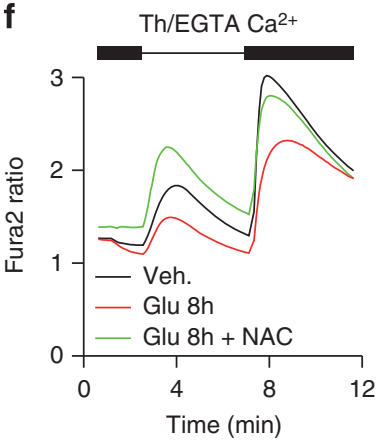

Figure 3 Prolonged oxidative stress reduces the $\mathrm{ER} \mathrm{Ca}^{2+}$ content and SOCE activity. (a) Application of pCPT-cGMP-induces cell death in HT22 cells. Cells were subjected to the indicated concentrations of pCPT-cGMP and viability was measured $16 \mathrm{~h}$ later with the cell-titer blue reagent. The graph shows the mean fluorescence values \pm S.E.M. of 11 replicate wells measured in two independent experiments plotted against glutamate concentration. (b) GCaMP5-based long-term $\mathrm{Ca}^{2+}$ live-cell imaging experiments reveals a pCPT-cGMP-induced cytosolic $\mathrm{Ca}^{2+}$ increase. Graphs show mean normalized GCaMP/RFP ratio of 10 wells each containing $>30$ cells obtained in two independent experiments. (c, $\mathbf{d}$ and $\mathbf{f}$ ) Fura2based SOCE measurements performed on a BD Pathway high content imaging system. Cells were either preincubated for $8 \mathrm{~h}$ with $2.5 \mathrm{~mm}$ glutamate, $2.5 \mathrm{~mm}$ glutamate together with $2 \mathrm{mM} \mathrm{NAC}$ or $2.5 \mathrm{~mm}$ pCPT-cGMP or supplemented with these substances directly at the onset of measurement as indicated. For measurement of SOCE after baseline recording in HBSS, ER Ca ${ }^{2+}$ stores were depleted by addition of $2 \mu \mathrm{m}$ thapsigargin in $2 \mathrm{~mm}$ EGTA, which was necessary to bind the $\mathrm{Ca}^{2+}$ ions of the HBSS buffer. After 6 min of store depletion, $5 \mathrm{~mm}$ of $\mathrm{Ca}^{2+}$ was added to overload the EGTA and allow SOCE. $\mathrm{Ca}^{2+}$ influx was recorded for additional $4 \mathrm{~min}$. Each trace represents the mean of five to six wells measured in three independent experiments. (e) Oxidative stress was induced in NAC or vehicle treated cells by application of indicated amounts of glutamate. Cell viability was quantitated $16 \mathrm{~h}$ after oxidative stress onset with the cell-titer blue reagent. The graph shows the mean \pm S.E.M. of nine replicate wells measured in three independent experiments plotted against glutamate concentration

and 2a) is relevant for the resistance against oxidative stress, we decided to identify the involved proteins. Resistance against oxidative stress in HT22R cells is mediated in part via
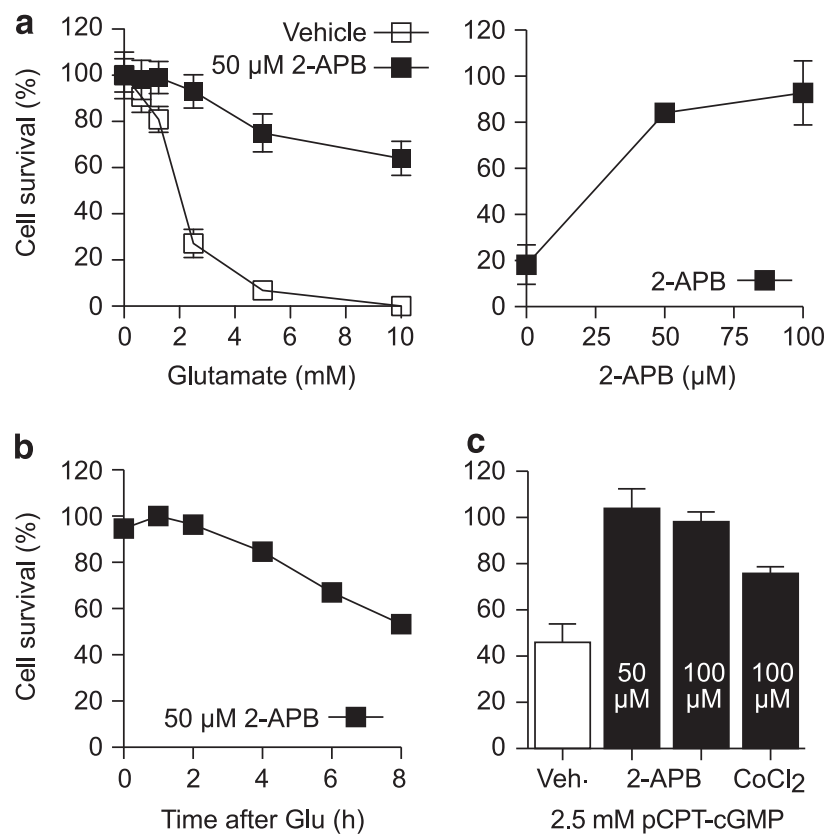

Figure 4 Pharmacological inhibition of SOCE protects against oxidative stress. (a) 2-APB or vehicle treated HT22S cells were subjected to oxidative stress by addition of the indicated amounts of glutamate. $16 \mathrm{~h}$ after stress onset, viability was quantitated with the CTB assay and normalized to controls without glutamate. Graphs show mean \pm S.E.M. of nine replicates obtained in three independent experiments. (a right panel) The indicated concentrations of 2-APB were added to HT22 cells treated with $5 \mathrm{~mm}$ of glutamate. $20 \mathrm{~h}$ after stress, onset viability was quantified with the MTT-viability assay. (b) 2-APB was added to glutamate (5 mM)treated HT22 cells at the indicated times after glutamate addition. After $20 \mathrm{~h}$, cell viability was quantified by the MTT-assay. (c) Cells were treated with the indicated concentrations of 2-APB, $\mathrm{CoCl}_{2}$ or vehicle and the MTT-assay was performed $20 \mathrm{~h}$ after addition of $2.5 \mathrm{mM}$ PCPT-cGMP

transcriptional regulation of protective proteins..$^{10,11,13} \mathrm{We}$ therefore hypothesized that the reduced SOCE observed in HT22R cells might be mediated via downregulation of components of the cellular SOCE machinery and analyzed the expression pattern of the SOCE-contributing proteins STIM1, STIM2 and ORAI1, as well the transient receptor potential melastatin 7 (TRPM7) channel, a $\mathrm{Ca}^{2+}$ permeable channel that is also blocked by 2-APB. ${ }^{20}$ The protein levels of STIM1 and STIM2 were equal in HT22S and R (Figure 5a and b). TRPM7 expression was analyzed by quantitative real-time-PCR (qRT-PCR) and also found to be the similar in both cell types (Figure 5c). Immunoblotting against ORAI1 produced a ladder of bands between 50 and $100 \mathrm{kDa}$, which may be due to protein modifications for example, glycosylation, differential splicing, ${ }^{21}$ dimerization or even tetramerization. ${ }^{22}$ We quantified the $50 \mathrm{kDa}$ band, which according to the datasheet of the antibody, should be ORAl1 and the more prominent $80 \mathrm{kDa}$ band. Both bands were significantly downregulated in HT22R cells (Figure $5 \mathrm{~d}$ ), consistent with the reduced SOCE activity in these cells. Downregulation of these bands by small inhibitory RNA (siRNA) transfection, which correlated with mRNA quantification by RT-PCR (Figure $6 \mathrm{~h}$ and i), also suggested specificity of the antibody used.

These results pointed to ORAl1 as being the protein that mediates the reduced SOCE observed in HT22R cells. To 

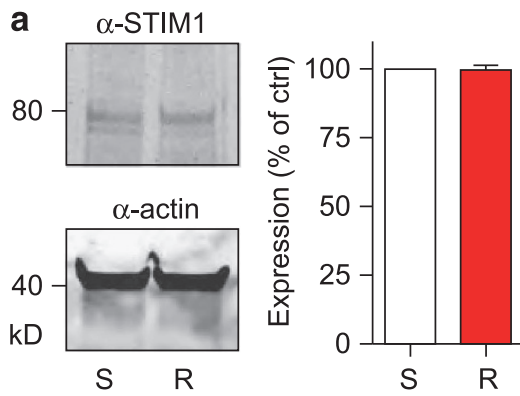

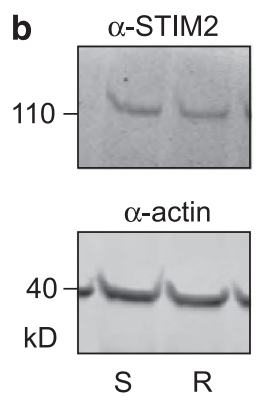

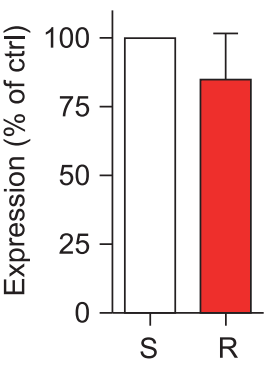

C
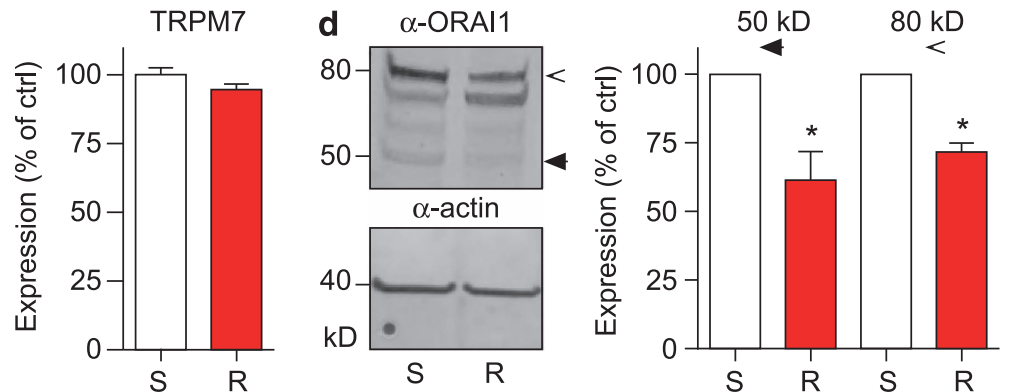

Figure 5 The SOCE Ca ${ }^{2+}$ influx channel ORAl1 is downregulated in glutamate-resistant HT22R cells. Expression analysis of key SOCE players and TRPM7 in HT22S and R cells. (a, b and d) Immunoblots were probed with antibodies against STIM1 (a) or 2 (b) and ORAl1 (d); GAPDH served as loading control and was simultaneously recorded with the protein of interest on the same membrane with the Licor infrared imaging system. Quantification of immunoblots was done with ImageJ software. Intensity values were normalized to HT22S samples and plotted in bar graphs as expression in percentage. (c) RT-PCR analysis of TRPM7 expression level in HT22S and R cells. The TRPM7 signal was normalized to the endogenous control hprt and plotted as mean expression level \pm S.E.M. in percentage. All graphs show the mean of at least three independently performed experiments. ${ }^{*} P<0.05$ using students $t$-test

complement this and to show that this downregulation is indeed involved in the resistance against oxidative stress observed in HT22R cells, we decided to knockdown the components of the SOCE machinery, STIM1, STIM2, ORAI1 and TRPM7 by siRNA and determine the susceptibility of the cells to oxidative glutamate toxicity.

Knockdown of ORAl1 protects from GSH depletion. We transfected HT22 cells with two different siRNAs against STIM1, STIM2 ORAI1 or TRPM7 and treated these cells with glutamate or L-buthionine-(S,R) sulfoximine (BSO), an inhibitor of the rate-limiting enzyme glutamate-cysteine ligase, $48 \mathrm{~h}$ after transfection. Successful knockdown was verified by RT-PCR analysis (Figure 6a, d, $f$ and $h$ ) and immunoblotting (Figure $6 \mathrm{~b}$ and $\mathrm{i}$ ). In line with the protein expression pattern observed in glutamate-resistant cells (Figure 5), knockdown of STIM1 or STIM2 induced no significant protective effect against GSH depletion elicited either by glutamate or BSO (Figure $6 c$ and e), whereas reduced ORAl1 levels strongly protected HT22 cells from oxidative stress (Figure 6j). Interestingly, siRNAmediated knockdown of TRPM7 in HT22S cells significantly increased susceptibility to oxidative glutamate toxicity (Figure 6g).

We conclude from these data that the protective effect of 2APB is unlikely to involve STIM1, STIM2 or TRPM7 inhibition, as reduction of these $\mathrm{Ca}^{2+}$ entry modulating proteins has either no or even the opposite effect of 2-APB. Rather, two lines of evidence suggest that ORAl1 is involved in oxidative stress-induced $\mathrm{Ca}^{2+}$ influx: ORAl1 is downregulated in glutamate-resistant cells and its knockdown protects glutamate-sensitive cells from cell death mediated by GSH depletion. We conclude that the protective effect of 2 -APB is mediated by the inhibition of ORAl1-mediated $\mathrm{Ca}^{2+}$ influx and that reduced expression of ORAI1 is involved in the resistance of HT22R cells against oxidative stress.

Knockdown of ORAl1 inhibits cytosolic calcium elevation during oxytosis. To directly investigate the contribution of ORAI 1 to the detrimental $\mathrm{Ca}^{2+}$ influx induced by glutamate, we continued with long-term $\mathrm{Ca}^{2+}$ live-cell imaging experiments to visualize the cytosolic $\mathrm{Ca}^{2+}$ rise during oxytosis. We transfected HT22 cells with the two different siRNAs against ORAl1 (Figure 6h, i) or nontargeting control-siRNA together with the $\mathrm{Ca}^{2+}$-sensor GCaMP5. Knockdown efficiency was controlled by RT-PCR (data not shown). $48 \mathrm{~h}$ after transfection, the cells were exposed to glutamate overnight and the cytosolic calcium signal was monitored in a high-content microscope equipped with an environmental chamber to keep the temperature and $\mathrm{CO}_{2}$ concentration constant. Two hours after glutamate addition, we started to acquire the GCaMP and the control RFP signal every $15 \mathrm{~min}$ for the next $14 \mathrm{~h}$ and calculated the GCaMP/RFP ratio. This showed that cells peak in a seemingly random manner between $5-16 \mathrm{~h}$ of glutamate exposure. After a sharp increase in GCaMP signal, the cells quickly lyse, which was visible through vigorous quenching of GCAMP and RFP fluorescence. A typical sequence of pictures is shown in Figure 7a. The appearance of these $\mathrm{Ca}^{2+}$ spikes was highly asynchronously spread over the whole-time frame (Figure 7a lower panel) and significantly less frequent in ORAI1 - siRNA-transfected cells compared 
a
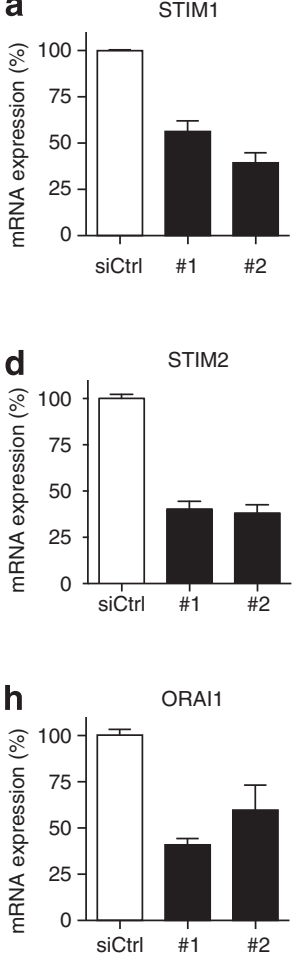

b
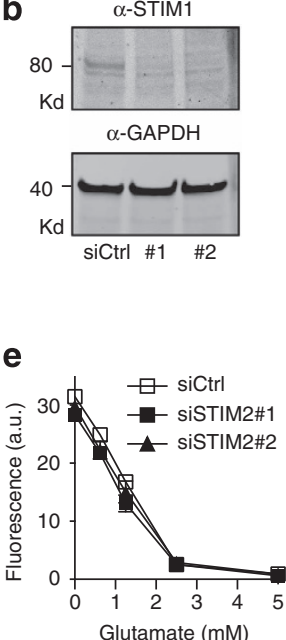

i

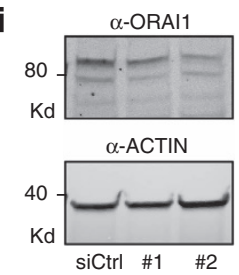

C
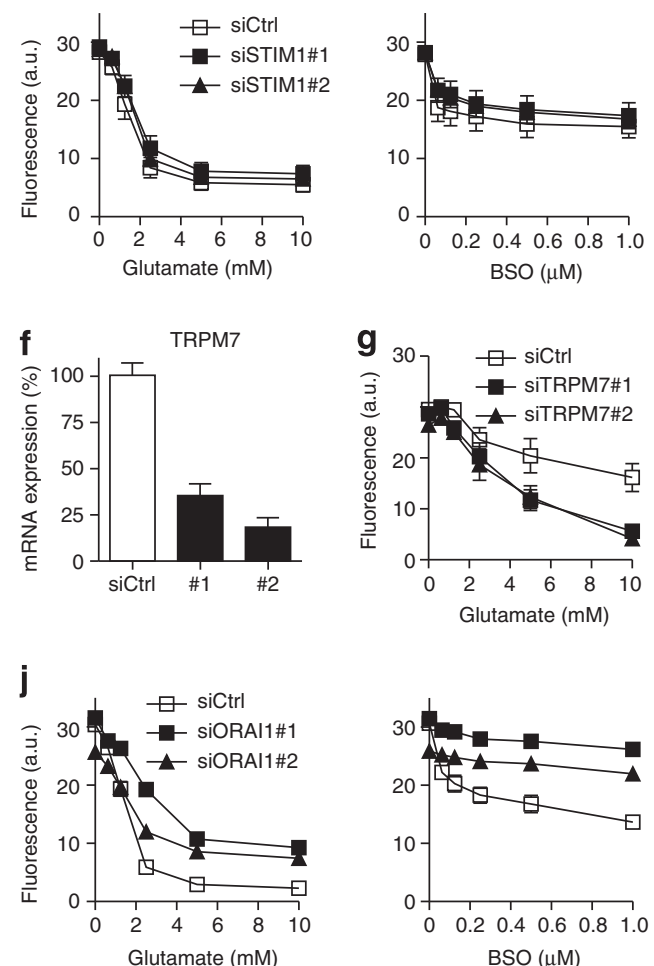

Figure 6 Knockdown of ORAl1 protects from GSH depletion. HT22S cells were transfected with two independent siRNAs against STIM1, STIM2, ORAl1 and TRPM7 or with non-targeting control siRNA and $48 \mathrm{~h}$ later subjected to cell survival experiments. (a, $\mathrm{d}, \mathrm{f}$ and $\mathrm{h}$ ) RT-PCR analyses of successful knockdown of STIM1 (a), STIM2 (d), ORAl1 (h) and TRPM7 (f). Signals of genes of interest were normalized to $h p r t$ and plotted as expression in percentage relative to control-siRNA transfected samples. (b and i) Verification of successful knockdown by immunoblot. The membranes were simultaneously probed with antibody against either STIM1 (b) or ORAl1 (i) and GAPDH respectively actin as loading control and analyzed with the Licor infrared imaging system. (c, e, $\mathbf{g}$ and $\mathbf{j})$ Survival experiments of siRNA-transfected cells. The indicated amounts of glutamate or BSO were added $48 \mathrm{~h}$ after transfection and viability was quantified with the CTB reagent $16 \mathrm{~h}$ after glutamate or BSO addition. The graphs show the mean fluorescence \pm S.E.M. of 15 replicates obtained in three independent experiments plotted against glutamate or BSO concentration

with scrambled siRNA-transfected cells (Figure 7b). We also treated ORAI1 siRNA-transfected cells with PCPT-cGMP and immediately recorded the cytosolic $\mathrm{Ca}^{2+}$ level with GCaMP5 as described above. Interestingly, pCPT-cGMP did not evoke $\mathrm{Ca}^{2+}$ peaks similar to glutamate, but instead induced a slow but prominent cytosolic $\mathrm{Ca}^{2+}$ influx (Figure $3 b$ ). Because the cytosolic $\mathrm{Ca}^{2+}$ increase induced by cell-permeable cGMP occurs with a steady increase, we calculated the slope of $\mathrm{Ca}^{2+}$ influx over the first $2 \mathrm{~h}$, which was significantly reduced by downregulation of ORAl1 (Figure 7c).

We conclude from these results that the SOCE-mediating channel protein ORAI1 significantly contributes to the detrimental $\mathrm{Ca}^{2+}$ influx elicited by cGMP or caused by oxidative stress in the course of GSH depletion.

\section{Discussion}

We conclude that ORAI1 channels has a major role in the detrimental $\mathrm{Ca}^{2+}$ influx caused by oxidative stress. We found that glutamate-resistant HT22R cells exhibit a reduction in SOCE and reduced amounts of ORAI1. Knockdown of ORAl1 protected against two different causes of GSH depletion; blocking cystine uptake with glutamate and inhibiting GSH synthesis with BSO. Pharmacological inhibition of SOCE using the well-established inhibitor 2-APB similarly protected against cell death induced by GSH depletion, but not against ER stress elicited with the $\mathrm{N}$-glycosylation inhibitor tunicamycin (not shown), which suggests that dysregulation of ORAl1 function is not a general aspect of cell death, despite the fact that glutamate-resistant cells are also resistant against ER stress as well as direct activation of the caspase cascade caused by overexpression of the apoptotic protein BAX. ${ }^{10}$ As 2-APB is a known inhibitor of $\mathrm{Ca}^{2+}$ influx, its cell survival-promoting action is most probably caused by its inhibitory action on the detrimental $\mathrm{Ca}^{2+}$ influx during oxidative stress. This hypothesis is strengthened by our time course experiments, which still showed a beneficial effect of 2APB when applied up to $8 \mathrm{~h}$ after glutamate addition, further pointing to a mechanism of action that interferes with late events in the cell death program such as $\mathrm{Ca}^{2+}$ influx. In addition, pCPT-cGMP-induced cell death was prevented by 2 $A P B$, and the observed effect was even stronger than that the general $\mathrm{Ca}^{2+}$ channel blocker $\mathrm{CoCl}_{2}$. Cytosolic elevation of cGMP through increased activity of soluble guanylate cyclases occurs very late in the cell death program induced by glutamate and is responsible for the opening of plasma membrane $\mathrm{Ca}^{2}$ channels allowing detrimental $\mathrm{Ca}^{2+}$ influx. ${ }^{7}$

The fact that knockdown of the upstream initiator of SOCE STIM1 had no effect, speaks against a dysregulation of the whole SOCE machinery and points instead to ORAI1 as the major culprit in these cells. When we investigated the effects of STIM1 and ORAI1 knockdown using the same siRNAs in 
a
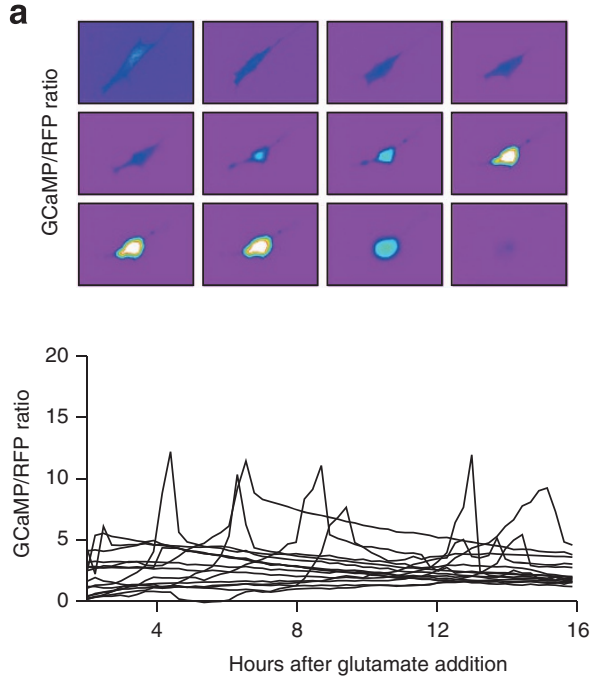

b

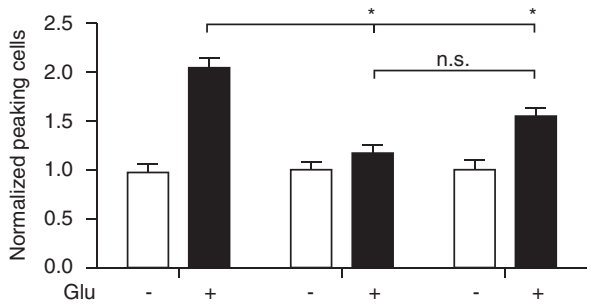

C

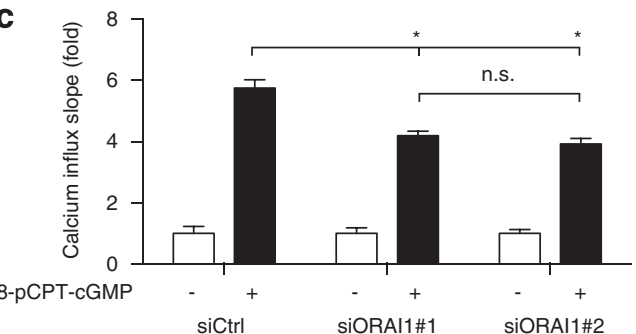

Figure 7 Knockdown of ORAl1 inhibits cytosolic calcium elevation during oxytosis. HT22S cells were transfected with two different siRNAs against ORAl1 or non-targeting siRNA as control together with a plasmid encoding the GCaMP5-IRES-RFP $\mathrm{Ca}^{2+}$ reporter gene and subjected to long-term live-cell imaging experiments to show the detrimental $\mathrm{Ca}^{2+}$ influx in the late phase of oxidative glutamate toxicity. (a) Typical GCaMP pictures of a control siRNA transfected cell under glutamate exposure. The cytosolic $\mathrm{Ca}^{2+}$ concentration stays low for several hours, but eventually rises quickly, leading to cell lysis assessed by quenching of the fluorescence signal. The lower panel shows sample traces from glutamate-treated cells transfected with control siRNA, which illustrate the asynchronous appearance of $\mathrm{Ca}^{2+}$-spikes. For reasons of clarity, only $\mathrm{Ca}^{2+}$-peak positive cells from one well are shown. (b) Analysis of long-term live-cell imaging of GCaMP-IRES-RFP and siRNA-transfected cells under glutamate exposure in comparison to vehicle treatment. For each picture, a GCaMP5/RFP ratio was calculated. To identify peaking cells, the maximal GCaMP/RFP ratio was divided by the average GCaMP/RFP ratio calculated over the whole measurement period. Each cell that reached a value above the average value of control siRNA transfected cells was counted as $\mathrm{Ca}^{2+}$-peak positive. The amount of $\mathrm{Ca}^{2+}$-peaking cells was normalized to vehicle control and plotted as a bar graph. The bars represent mean \pm S.E.M. of 15 replicate wells measured in three independent experiments. (c) Analysis of the increase in the GCaMP/RFP ratio in pCPT-cGMP treated HT22 cells transfected with ORAl1 or control siRNA. The slope was normalized to vehicle and plotted as mean \pm S.E.M. in bar graphs. Two independent experiments were performed containing $>150$ cells for each condition. N.S., not significant, ${ }^{*} P<0.05$ by ANOVA with Tukey's post hoc test

mouse embryonic fibroblasts, we observed a detrimental effect for both transcripts, which suggests cell type-specific differences. ${ }^{23}$ The protection conferred by ORAl1 knockdown in HT22 cells is in line with the recent observation that CD4positive $T$ cells from mice lacking ORAl1 robustly proliferate despite repetitive stimulations, and are strongly resistant against stimulation-induced cell death. ${ }^{24}$ Our results suggest that ORAl1-mediated $\mathrm{Ca}^{2+}$ influx causes cytotoxic $\mathrm{Ca}^{2+}$ overload late in the course of oxidative stress-induced cell death. To prove this hypothesis, we performed long-term live-cell imaging of glutamate challenged cells transfected with a genetically-encoded cytosolic $\mathrm{Ca}^{2+}$ sensor. The direct observation of cytosolic calcium dynamics revealed a strong but short-lived calcium rise directly preceding cell death, which appeared completely asynchronously in the cell population within a time frame of $5-16 \mathrm{~h}$ after the addition of glutamate. This perfectly resembles the time course of 2-APB protection. When applied $8 \mathrm{~h}$ after glutamate (Figure $4 \mathrm{~b}$ ), $50 \%$ of the cells were still rescued, which can be explained by the apparently random distribution of the final $\mathrm{Ca}^{2+}$ rise. Cells that have already opened their gates for external $\mathrm{Ca}^{2+}$ are irrecoverable, but those that have not induced $\mathrm{Ca}^{2+}$ influx at the time of 2-APB addition can still survive by the blockage of $\mathrm{Ca}^{2+}$ channels.

Our quantification of $\mathrm{Ca}^{2+}$ dynamics cells during glutamate or pCPT-cGMP treatment revealed a direct impact of ORAI1 expression levels on the $\mathrm{Ca}^{2+}$ influx after induction of the cell death program, which is perfectly in line with the protective effect of ORAl1 knockdown in the survival assays. Direct $\mathrm{Ca}^{2+}$ imaging over the whole-time course of oxidative glutamate toxicity also disclosed the time lag between cytosolic $\mathrm{Ca}^{2+}$ elevation and cell death. We demonstrated that the cytosolic $\mathrm{Ca}^{2+}$ rise directly precedes cell death, visible by disintegration of the plasma membrane and strong fluorescence quenching due to loss of GCaMP5. This short time period between the cytosolic $\mathrm{Ca}^{2+}$ signal and cell death suggests a direct detrimental role and renders further signaling events unlikely.

Although we showed that intracellular cGMP contributes to $\mathrm{Ca}^{2+}$ channel opening, it remains unclear whether it directly causes the opening of ORAl1 channels in endogenous oxidative stress or whether additional factors are needed. It is possible that the reduced amounts of STIM1 in siRNAtransfected cells (Figure 6a, b) are still sufficient to open ORAI1 channels during oxidative stress or that STIM2 can substitute for STIM1 under these conditions. Furthermore, ORAI1 might not only be activated by STIM1, but also through other factors such as the $\mathrm{Ca}^{2+}$-independent phospholipase $\mathrm{A}_{2}$ (iPLA2 $\left.\beta\right) .{ }^{25}$ Also a direct influence of the cellular redox state on ORAI1 activity seems possible. One study revealed that $\mathrm{H}_{2} \mathrm{O}_{2}$ enhanced $\mathrm{I}_{\mathrm{CRAC}}$-mediated $\mathrm{Ca}^{2+}$ influx (the electrophysiological equivalent of SOCE) through activation of IP3 receptors and subsequent store depletion without direct effect on ORAI1. ${ }^{26}$ SERCA pumps in contrast were shown to be inhibited by $\mathrm{H}_{2} \mathrm{O}_{2} \cdot{ }^{27}$ One might speculate that during glutamate-induced oxidative stress, SERCA is inhibited and IP3 receptors activated, which eventually results in $\mathrm{ER} \mathrm{Ca}^{2+}$ store depletion triggering ORAl1-mediated detrimental $\mathrm{Ca}^{2+}$ influx. This would be in line with our results, where $8 \mathrm{~h}$ of glutamate treatment resulted in a reduction of the 
thapsigargin-releasable $\mathrm{Ca}^{2+}$ pool, implying reduced SERCA activity or increased ER $\mathrm{Ca}^{2+}$ efflux. Others, however, suggested a direct inhibitory effect of $\mathrm{H}_{2} \mathrm{O}_{2}$ on ORAl1mediated $\mathrm{Ca}^{2+}$ influx induced by store depletion with thapsigargin, which was explained by the oxidation of cysteine residues located in an extracellular loop of ORAI1. ${ }^{28}$ This could be interpreted as an attempt to withstand the cytotoxic $\mathrm{Ca}^{2+}$-mediated by ORAI1.

We conclude from the presented data that dysregulated ORAl1-mediated $\mathrm{Ca}^{2+}$ influx contributes in a significant manner to programmed cell death induced by GSH depletion in neuronal cells. As this detrimental $\mathrm{Ca}^{2+}$ influx occurs late in the course of the cell death program, it might be amenable to therapeutic intervention in acute diseases where oxidative stress have a role.

\section{Materials and Methods}

Cell culture. The glutamate-resistant HT22R cell line was generated from the parental mouse hippocampal cell line HT22 as previously described..$^{19}$ Both cells lines, HT22S and HT22R, were cultured in DMEM high glucose (PAA Laboratories, Pasching, Austria ) supplemented with $5 \%$ fetal calf serum (Thermo Fisher Scientific, Waltham, MA, USA) and $100 \mathrm{U}$ per ml penicillin and $100 \mu \mathrm{g}$ per $\mathrm{ml}$ streptomycin (Gibco, Darmstadt, Germany) in a humidified incubator with $5 \%$ $\mathrm{CO}_{2}$ and $95 \%$ air.

Cell survival assays. HT22 cells were seeded in 96-well plates at a density of $5000 \mathrm{cells} / \mathrm{well}$ and $24 \mathrm{~h}$ later subjected to cell death experiments. siRNAtransfected cells were seeded $24 \mathrm{~h}$ post transfection. Pharmacological agents were added together with, or at specified time periods after, induction of cell death by the indicated drugs. Cell viability was quantified $16-24 \mathrm{~h}$ after stress onset with the cell titer blue (CTB) reagent (Promega, Madison, WI, USA). Alternatively, in some experiments, the 3-(4,5-Dimethylthiazol-2-yl)-2,5-diphenyltetrazolium bromide (MTT) assay was used. ${ }^{19}$ For CTB fluorescence, emission was measured at $590 \mathrm{~nm}$ after excitation at $562 \mathrm{~nm}$ using a GENios Pro microplate reader (Tecan, Männedorf, Switzerland); for the MTT-assay, absorption was measured at $570 \mathrm{~nm}$.

Calcium live-cell imaging. Fura2 $\mathrm{Ca}^{2+}$ imaging experiments were performed on a BD Pathway 855 High Content Imaging System (BD Biosciences, Heidelberg, Germany). The cells were seeded in 96-well imaging plate (BD Bioscience) the day before the experiment in a density of $5000 \mathrm{cells} / \mathrm{well}$ and loaded with $5 \mu$ m Fura2-AM (Molecular Probes, Darmstadt, Germany) in HBSS for $30 \mathrm{~min}$ before the experiment. Measurement was performed with excitation at 340 and $380 \mathrm{~nm}$ for ratiometric analysis and pictures were taken with a delay of $5 \mathrm{~s}$. For comparison of SOCE in HT22S and HT22R, cells were seeded on $12 \mathrm{~mm}$ coverslips at a density of 100000 /well, loaded with Fura2-AM and placed into a flow chamber. The measurement was performed using an Olympus IX81 fluorescence microscope with the cell$\wedge R$ imaging software. Images were acquired at 340 and $380 \mathrm{~nm}$ excitation and the ratio was calculated every $5 \mathrm{~s}$ for every single cell. After $1 \mathrm{~min}$ of baseline recording, ER-calcium stores were depleted in EGTA buffer $\left(\mathrm{Ca}^{2+}\right.$-free HBSS supplemented with $0.5 \mathrm{~mm}$ EGTA, $20 \mathrm{~mm}$ HEPES, $1 \mathrm{~mm} \mathrm{MgCl} 2$ and $1 \mathrm{~g}$ per I Glucose) with $2 \mu \mathrm{m}$ thapsigargin for $6 \mathrm{~min}$ and subsequently $\mathrm{Ca}^{2+}$ was readded by changing the buffer to HBSS.

Electrophysiology. We used whole-cell patch clamping to measure currents through ion channels of single cells. Whole-cell recordings were performed using an Axopatch 200B patch clamp amplifier (Axon Instruments, Sunnyvale, CA, USA) and digitized with Digidata 1332 (Axon Instruments). For SOC channels recordings the pipette solution contained (in mM) $120 \mathrm{CsCl}, 5$ Bapta-Na, $30 \mathrm{Cs}$-HEPES pH 7.3, $1.5 \mathrm{MgCl}_{2}, 4 \mathrm{Na}_{2} \mathrm{ATP}, 0.4 \mathrm{Na}_{2} \mathrm{GTP}$ and $1.6 \mathrm{CaCl}_{2}$ (pCa 7.0). Extracellular solution contained (in mM) 140 NMDG-Asp, $10 \mathrm{BaCl}_{2}, 10$ Cs-HEPES, pH 7.3. During recording the currents were sampled at $5 \mathrm{kHz}$ and filtered digitally at $500 \mathrm{~Hz}$, the pClamp9 software (Axon Instruments) was used for data acquisition and analysis. In all whole-cell experiments the holding potential was $-40 \mathrm{mV}$, periodically (once every $5 \mathrm{~s}$ ) the membrane potential was stepped to $-100 \mathrm{mV}$ (for $30 \mathrm{~ms}$ ) and a $200 \mathrm{~ms}$ voltage ramp to $+100 \mathrm{mV}$ was applied. Currents were evoked by application of $1 \mu \mathrm{m}$ thapsigargin (Sigma, Munich, Germany) in external solution, which was added to the bath perfusion. The traces recorded before current activation were used as templates for leak subtraction. Whole-cell currents were normalized to the cell capacitance. The mean value of cell capacitance was $19 \mathrm{pF} \pm 5$ (total number of experiments $n=20$ ).

Quantitative RT-PCR. Whole-cellular RNA was isolated using ZR RNA MiniPrep Kit (Zymo, Irvine, CA, USA) and transcribed into CDNA with the High Capacity cDNA Reverse Transcription Kit (Applied Biosystems, Darmstadt, Germany). RT-PCR analysis was performed on a 7500 Fast cycler (Applied Biosystems) with FAST BLUE qPCR MasterMix (Eurogentec, Cologne, Germany) running the 7500 standard program. Primers and Probes for STIM1 and 2, ORAl1 and TRPM7 were designed by the universal probe library assay design center (Roche, Mannheim, Germany) and the hypoxanthine-phosphoribosyltransferase gene (HPRT) served as an endogenous control with individually designed primers and probe purchased from MWG (Ebersberg, Germany).

siRNA transfections. Flexitube siRNAs against ORAI1, STIM1, STIM2 and TRPM7 were purchased from Qiagen (Hilden, Germany) (no.SI00972251, no.SI00972272, no.Sl1435623, no.SI1435637, no.SI01435665, no.SI01435672, no.SI02694727 and no.SI02742663) and transfected into cells with Lipofectamine RNAiMAX (Invitrogen, Darmstadt, Germany) when cells were grown to $70-80 \%$ confluence in six-well plates. Successful knockdown was verified by RT-PCR or immunoblot.

Immunoblotting. Cells were lysed in ice-cold RIPA buffer (Thermo Fisher Scientific) containing mini complete protease inhibitor cocktail (Roche) and centrifuged for $30 \mathrm{~min}$ at $16000 \mathrm{~g}$. The supernatants were separated on $8-16 \%$ polyacrylamide gels (Thermo Fisher Scientific), transferred onto nitrocellulose membranes with the iBlot System (Invitrogen, Darmstadt, Germany) and blocked in $3 \%$ nonfat dry milk in phosphate-buffered saline containing $0.5 \%$ Tween-20 (PBS-T) for $1 \mathrm{~h}$ at room temperature before overnight incubation with primary antibodies against STIM1 (BD Biosciences, no.610955 1:250), STIM2 (Cell Signaling, Danvers, MA, USA, no.4917 1:1000), ORAl1, Alomone Jerusalem, Israel, no.ACC-062 1:500), Actin (Millipore, Billerica, MA, USA, no.MAB1501 $1: 5000$ ) or GAPDH (Cell Signaling, no.2118 1:5000) followed by $\alpha$-mouse (respectively rabbit) $\operatorname{lgG}(\mathrm{Fc})$ infrared fluorescence-conjugated secondary antibody (Licor, Lincoln, NE, USA, 1:30000). The membranes were scanned for infrared fluorescence at 680 or $800 \mathrm{~nm}$ using the Odyssey system (Licor) and the signal was analyzed quantitatively with the image-processing software ImageJ (http:// rsbweb.nih.gov(iij).

Long-term calcium live-cell imaging. GCaMP5 was a kind gift of Douglas S. Kim (Howard Hughes Medical Institute, Ashburn) and subcloned with EcoRI and Notl into the IRES-RFP-containing vector PB531A-1 (System Biosciences, Mountain View, CA, USA). Cells were transfected with GCaMP5IRES-RFP together with two different siRNAs against ORAl1 or non-targeting control-siRNA with Lipofectamine 2000 (Invitrogen) in six-well plates and $24 \mathrm{~h}$ later transferred to 96-well imaging plates (BD Biosciences) at a density of 5000 cells/ well. For calcium imaging, phenol red containing medium was replaced by colorless DMEM (PAA Laboratories, Pasching, Austria). Two hours before the onset of measurement, $25 \mathrm{~mm}$ glutamate was added to the cells, control wells were supplemented with vehicle and the plate was incubated in the BD Pathway 855 at $37^{\circ} \mathrm{C}$ with $5 \% \mathrm{CO} 2$ and $95 \%$ air. For pCPT-cGMP-induced $\mathrm{Ca}^{2+}$ influx, measurement was started immediately after addition of $2.5 \mathrm{~mm}$ pCPT-cGMP. Cytosolic calcium was monitored by GCaMP5 and normalized to RFP fluorescence; pictures were taken every $15 \mathrm{~min}$. For analysis of glutamateinduced $\mathrm{Ca}^{2+}$ peaks, the maximal ratio was divided by the average ratio and a threshold was defined by the mean of control-siRNA transfected cells. Every cell above that threshold was counted as $\mathrm{Ca}^{2+}$ peak positive. For analysis of pCPTcGMP-induced $\mathrm{Ca}^{2+}$ influx, the slope of $\mathrm{Ca}^{2+}$ increase was calculated over the first $2 \mathrm{~h}$ of the measurement.

Statistical analysis. Data were analyzed as mean \pm S.E.M. and the statistical significance assessed using two-tailed $t$-tests or analysis of variance (ANOVA) with Tukey's Multiple Comparison Test as indicated.

\section{Conflict of Interest}

The authors declare no conflict of interest. 
Acknowledgements. We thank Andrea Issberner and Caroline Sladek for excellent technical support and Christie Dietz for proofreading. This project was funded by Dr. Kurt and Irmgard Meister-Stiftung (AM), 'Molecular and Cellular Biology' RAS (EK), the OPTEC LLC (MR), and TargetSOCE (AM and EK).

1. Dringen R. Metabolism and functions of glutathione in brain. Prog Neurobiol 2000; 62 649-671.

2. Dixon SJ, Lemberg KM, Lamprecht MR, Skouta R, Zaitsev EM, Gleason CE et al. Ferroptosis: an iron-dependent form of nonapoptotic cell death. Cell 2012; 149: 1060-1072.

3. Albrecht P, Lewerenz J, Dittmer S, Noack R, Maher P, Methner A et al. Mechanisms of oxidative glutamate toxicity: the glutamate/cystine antiporter system $\mathrm{xc}$ - as a neuroprotective drug target. CNS Neurol Disord Drug Targets 2010; 9: 373-382.

4. Tan S, Sagara $Y$, Liu Y, Maher P, Schubert D. The regulation of reactive oxygen species production during programmed cell death. J Cell Biol 1998; 141: 1423-1432.

5. Li Y, Maher P, Schubert D. A role for 12-lipoxygenase in nerve cell death caused by glutathione depletion. Neuron 1997; 19: 453-463.

6. Pallast S, Arai K, Wang X, Lo EH, van Leyen K. 12/15-Lipoxygenase targets neuronal mitochondria under oxidative stress. J Neurochem 2009; 111: 882-889

7. Li Y, Maher P, Schubert D. Requirement for cGMP in nerve cell death caused by glutathione depletion. J Cell Biol 1997; 139: 1317-1324.

8. Davis JB, Maher P. Protein kinase $C$ activation inhibits glutamate-induced cytotoxicity in a neuronal cell line. Brain Res 1994; 652: 169-173.

9. Murphy TH, Miyamoto M, Sastre A, Schnaar RL, Coyle JT. Glutamate toxicity in a neuronal cell line involves inhibition of cystine transport leading to oxidative stress. Neuron 1989; 2 : 1547-1558

10. Dittmer S, Sahin M, Pantlen A, Saxena A, Toutzaris D, Pina AL et al. The constitutively active orphan G-protein-coupled receptor GPR39 protects from cell death by increasing secretion of pigment epithelium-derived growth factor. J Biol Chem 2008; 283: 7074-7081.

11. Noack R, Frede S, Albrecht P, Henke N, Pfeiffer A, Knoll K et al. Charcot-Marie-Tooth disease CMT4A: GDAP1 increases cellular glutathione and the mitochondrial membrane potential. Hum Mol Genet 2012; 21: 150-162.

12. Sahin M, Saxena A, Joost $P$, Lewerenz J, Methner A. Induction of Bcl-2 by functional regulation of G-protein coupled receptors protects from oxidative glutamate toxicity by increasing glutathione. Free Radic Res 2006; 40: 1113-1123.

13. Toutzaris D, Lewerenz J, Albrecht P, Jensen LT, Letz J, Geerts A et al. A novel giant peroxisomal superoxide dismutase motif-containing protein. Free Radic Biol Med 2010; 48: $811-820$.

14. Roos J, DiGregorio PJ, Yeromin AV, Ohlsen K, Lioudyno M, Zhang S et al. STIM1, an essential and conserved component of store-operated $\mathrm{Ca} 2+$ channel function. J Cell Biol 2005; 169: 435-445

15. Liou J, Kim ML, Heo WD, Jones JT, Myers JW, Ferrell JE Jr et al. STIM is a Ca2 + senso essential for $\mathrm{Ca} 2$ + -store-depletion-triggered Ca2 + influx. Curr Biol 2005; 15: 1235-1241.
16. Zhang SL, Yeromin AV, Zhang XH, Yu Y, Safrina O, Penna A et al. Genome-wide RNAi screen of $\mathrm{Ca}(2+)$ influx identifies genes that regulate $\mathrm{Ca}(2+)$ release-activated $\mathrm{Ca}(2+)$ channel activity. Proc Natl Acad Sci USA 2006; 103: 9357-9362.

17. Prakriya M, Feske S, Gwack $Y$, Srikanth $S$, Rao A, Hogan PG et al. Orai1 is an essential pore subunit of the CRAC channel. Nature 2006; 443: 230-233.

18. Mignen $\mathrm{O}$, Thompson JL, Shuttleworth TJ. Orai1 subunit stoichiometry of the mammalian CRAC channel pore. J Physiol (Lond) 2008; 586: 419-425.

19. Lewerenz J, Klein M, Methner A. Cooperative action of glutamate transporters and cystine/ glutamate antiporter system Xc- protects from oxidative glutamate toxicity. J Neurochem 2006; 98: 916-925.

20. Li M, Jiang J, Yue L. Functional characterization of homo- and heteromeric channel kinases TRPM6 and TRPM7. J Gen Physiol 2006; 127: 525-537.

21. Fukushima M, Tomita T, Janoshazi A, Putney JW. Alternative translation initiation gives rise to two isoforms of orai1 with distinct plasma membrane mobilities. J Cell Sci 2012; 125: 4354-4361.

22. Penna A, Demuro A, Yeromin AV, Zhang SL, Safrina O, Parker I et al. The CRAC channel consists of a tetramer formed by Stim-induced dimerization of Orai dimers. Nature 2008; 456: $116-120$.

23. Henke N, Albrecht P, Pfeiffer A, Toutzaris D, Zanger K, Methner A et al. Stromal interaction molecule 1 (STIM1) is involved in the regulation of mitochondrial shape and bioenergetics and plays a role in oxidative stress. J Biol Chem 2012; 287: 42042-42052.

24. Kim KD, Srikanth S, Yee MK, Mock DC, Lawson GW, Gwack Y et al. ORAl1 deficiency impairs activated $t$ cell death and enhances T cell survival. J Immunol 2011; 187: $3620-3630$.

25. Bolotina VM. Orai, STIM1 and iPLA2beta: a view from a different perspective. J Physiol (Lond) 2008; 586: 3035-3042.

26. Grupe M, Myers G, Penner R, Fleig A. Activation of store-operated ICRAC by hydrogen peroxide. Cell Calcium 2010; 48: 1-9.

27. Kuster GM, Lancel S, Zhang J, Communal C, Trucillo MP, Lim CC et al. Redox-mediated reciprocal regulation of SERCA and $\mathrm{Na}+-\mathrm{Ca} 2+$ exchanger contributes to sarcoplasmic reticulum $\mathrm{Ca} 2+$ depletion in cardiac myocytes. Free Radic Biol Med 2010; 48: 1182-1187.

28. Bogeski I, Kummerow C, Al-Ansary D, Schwarz EC, Koehler R, Kozai D et al. Differential redox regulation of orai ion channels: a mechanism to tune cellular calcium signaling. Sci Signal 2010; 3: ra24.

Cell Death and Disease is an open-access journal published by Nature Publishing Group. This work is licensed under the Creative Commons Attribution-NonCommercial-No Derivative Works 3.0 Unported License. To view a copy of this license, visit http://creativecommons.org/licenses/by-nc-nd/3.0/ 